\title{
Standardless EDS Quantitative Analysis at High Tilt Angles
}

\author{
A.O. Sandborg*, R.A. Anderhalt*, J. M. Dijkstra**, R. B. Shen* \\ *EDAX, Inc. 85 McKee Drive, Mahwah, NJ 07430 \\ *EDAX Europe, Ringbaan Noord 103, 5046 AA Tilburg, The Netherlands
}

Elemental information can greatly aid the phase identification of materials from diffraction patterns acquired on an EBSD system. Searching a crystallographic database can be faster and less ambiguous when elemental information is included with the crystallographic data. The data is collected at high sample tilt angles of 60 to 70 degrees. While this is favorable for reducing the amount of absorption of lower energy xrays leaving the sample, the matrix correction methods used to correct for absorption are not accurate when applied at such high tilt angles. Also, because of the high tilt angles used, it is not convenient to use standards in the quantitative analysis procedure.

The difficulties arise primarily in the application of an absorption correction. The depth distibution of the $\mathrm{x}-$ rays changes shape quite radically at high tilt angles. The absorption corrections at 0 or low angle tilts use assumptions on this shape which are not adequate at tilts of $>50$ degrees. We have evaluated a ZAF method that assumes a square model for the depth distribution, and also a PhiRhoZ model which assumes a gaussian shape [1,2]. Surprisingly, these models perform similarly, under correcting for absorption.

The sample measured is an Al-Ni alloy of nearly 50/50 \% Atomic percent composition. The Al weight concentration is $31.5 \%$. This matrix will have a substantial absorption correction when analyzed at 15 to 20 $\mathrm{kV}$, which would be typical conditions for achieving optimal EBSD patterns. This sample was measured in several SEM with differing geometries of the electron beam to sample to detector. Figure 1 shows the Al wt $\%$ as a function of sample tilt angle using a ZAF method with the Bishop square model of absorption correction and a PhiRhoZ model. The Al Wt\% declines with a tilt angle of higher than 30 degrees.

Correction for absorption has been the focus of researchers since the early days of microprobe analysis. Once the nature of the PhiRhoZ curve was understood, attempts have been made to model its shape in order to properly correct for absorption. These models are successful at low sample tilt angles, however the shape of the PhiRhoZ curve changes radically at high tilt angles causing the type of results shown above. Figure 2 shows Monte Carlo simulations of the PhiRhoZ distributions of $\mathrm{Al}$ in AlNi with tilt angles of 0 to 70 degrees. A simple modification of Bishop to his square model to correct for tilt was incorporated into the absorption correction [3]. The results of this change are shown in Figure 3. At tilts $>35$ degrees, this correction gives a clear improvement over uncorrected results. The results at high tilt are quite satisfactory for searching a diffraction database for the purpose of identifying a phase using chemistry and diffraction data.

Further modifications to PhiRhoZ and ZAF matrix corrections will be explored to allow a comprehensive comparison of the methods.

References:

[1] Love G., and Scott, V. D., J. Phys. D:Appl. Phys., 11 (1978) 1369

[2] Bastin, G. F. et al, Scanning, 5 (1986) 45

[3] Bishop, H. E., J. Phys. D:Appl. Phys., 1 (1968) 673 


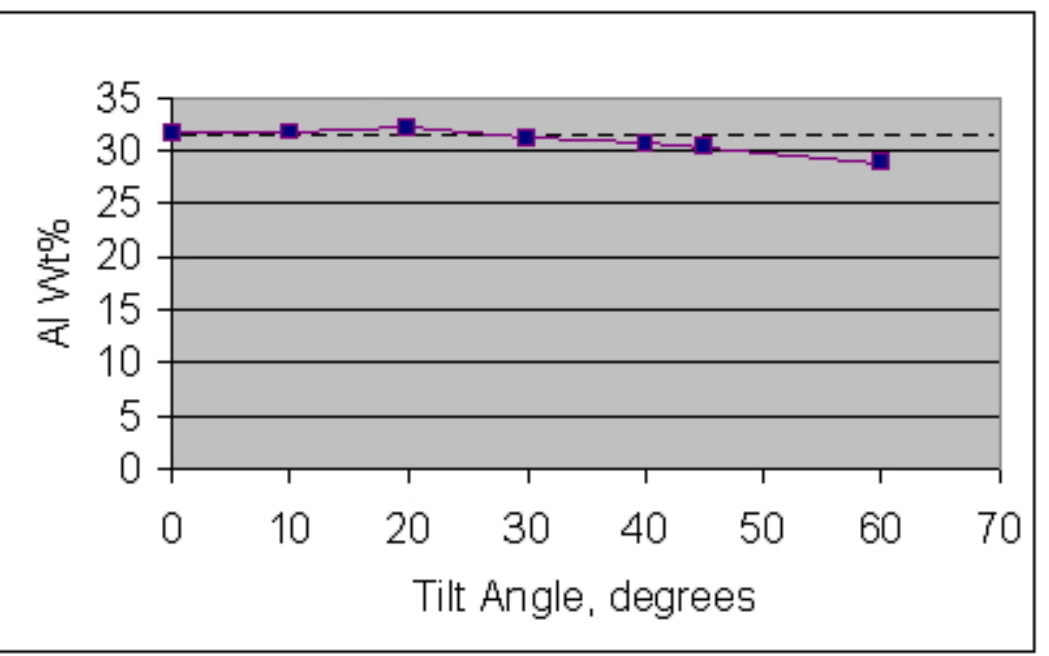

Figure 1. Al Wt\% in AINi using an unmodified ZAF correction

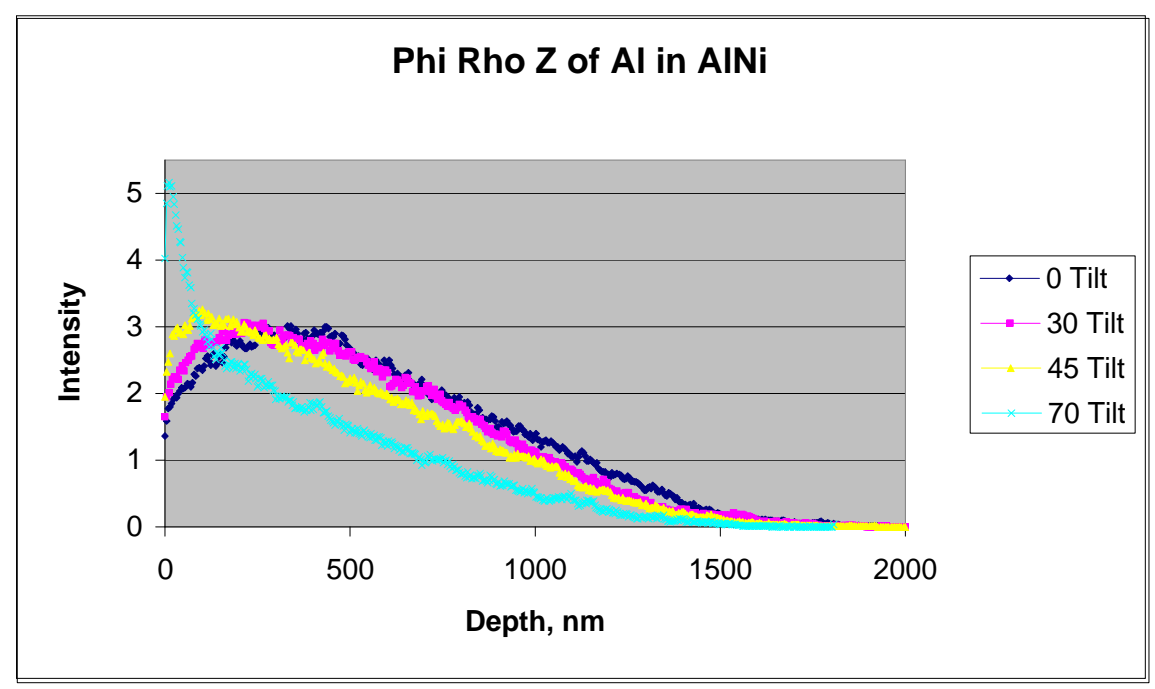

Figure 2. Phi Rho $\mathrm{Z}$ curves of $\mathrm{Al}$ in $\mathrm{AINi}$ at varying sample tilts

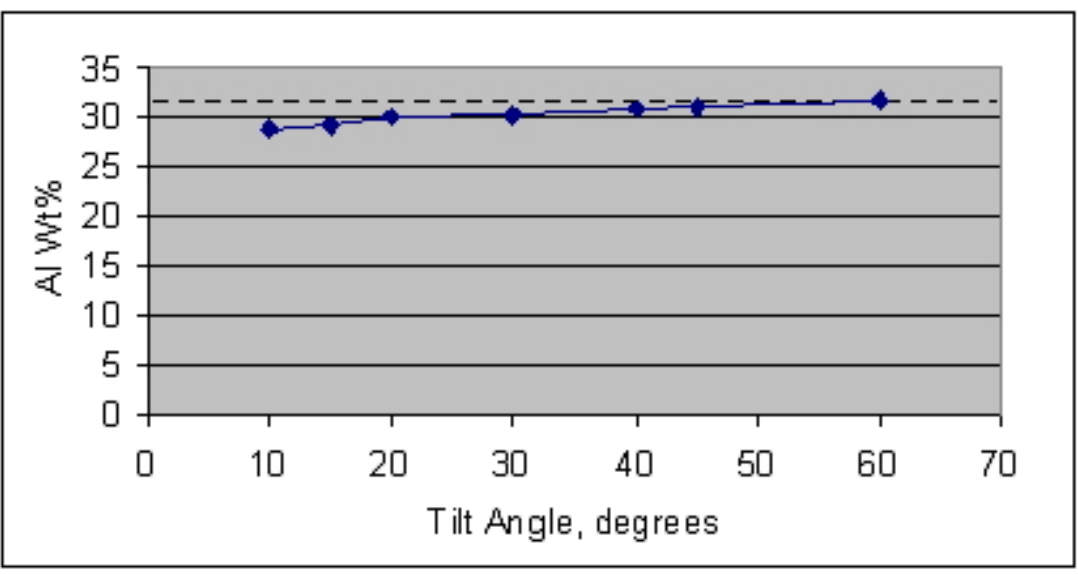

Figure 3. Al Wt\% in AlNi using a modified ZAF correction 